\title{
Hipoglicemia como Fator Complicador no Tratamento do Diabetes Melito Tipo 1
}

revisão

MÁRCIA NERY

Hospital das Clínicas da Faculdade de Medicina da Universidade de São Paulo (HCFMUSP), SP, Brasil.

\section{RESUMO}

Os portadores de diabetes melito tipo 1 têm, com freqüência, episódios de hipoglicemia durante a insulinoterapia, que, além do desconforto e de proporcionar situações constrangedoras no dia-a-dia, impedem a obtenção do controle glicêmico ideal. Mais ainda, hipoglicemias induzem deficiente mecanismo de contra-regulação em episódio posterior, com diminuição de liberação de adrenalina e dos sintomas de alarme, estabelecendo a síndrome de hipoglicemia associada à insuficiência autonômica. A ocorrência de hipoglicemias durante algumas atividades de risco, em especial a direção veicular, pode resultar acidentes com o paciente e terceiros, além de lesão de propriedade, motivo pelo qual pessoas com diabetes devem ser orientadas quanto aos cuidados na direção de veículos. Em geral, a recuperação neurológica é total após a correção de coma hipoglicêmico. No entanto, quando esses episódios são repetitivos, especialmente em crianças, podem ter como conseqüência distúrbios cognitivos definitivos. A reversão de quadros de hipoglicemia sem sinal de alerta é difícil, devendo-se evitar meticulosamente sua ocorrência, adequando o tratamento, os alvos glicêmicos, utilizando a monitoração domiciliar e fazendo treinamento para o reconhecimento precoce de hipoglicemias. (Arq Bras Endocrinol Metab 2008;52/2:288-298)

Descritores: Diabetes do tipo 1; Hipoglicemia; Contra-regulação; Insuficiência autonômica; Distúrbios cognitivos

\begin{abstract}
Hypoglycemia as a Limiting Factor in the Management of Type 1 Diabetes.

Type 1 diabetic patients frequently present hypoglycemic episodes during their insulinotherapy, which, besides the discomfort and constrains does not allow the ideal glycemic control. Further, hypoglycemic events lead to the deficiency of the counter-regulation mechanisms in the subsequent episode, with a decrease in the release of epinephrine and the symptoms of warming, with great risk of severe hypoglycemia. The occurrence of hypoglycemia during some risky activities, specially driving, could result in accidents with the patient and /or third parts including property damage, stressing here the need to advise diabetics against having the necessary caution wheli driving. Generally the connective recovery is total after correcting a hypoglycemic coma. However when these episodes are repetitive, particularly in children, they could result in definitive cognitive disturbances. Hypoglycemic events without a warning signal (hypoglycemic unawareness) are difficult to reverse, thus it is necessary to prevent their occurrence, adjusting the treatment with glycemic targets, using continuous glucose monitoring at home and teaching them how to have an early recognition of hypoglycemia. (Arq Bras Endocrinol Metab 2008;52/2:288-298)
\end{abstract}

Recebido em 22/1 1/2007 Aceito em 03/12/2007
Keywords: Type 1 diabetes; Treatment; Hypoglycemia; Counter-regulation; Autonomic failure; Cognitive dysfunction 


\section{INTRODUÇÃO}

S HIPOGLICEMIAS IATROGÊNICAS acometem até 90\%

das pessoas tratadas com insulina, sendo o fator limitante para a adequação da terapêutica; pode-se dizer que são uma barreira bastante difícil de transpor para a obtenção de controle glicêmico adequado (1).

Como observado em vários estudos, a intensificação do controle metabólico aumentou a freqüência de hipoglicemia. Os portadores de diabetes melito tipo 1 (DMl) podem ter valores glicêmicos entre 50 e 60 em até $10 \%$ do tempo; hipoglicemias sintomáticas várias vezes por semana. Hipoglicemias graves com perda de consciência, convulsões, coma a cada um ou dois $\operatorname{anos}(2)$ ou de 0,20 a 0,601 eventos por pessoa/ ano $(1-3)$.

No entanto, o risco de hipoglicemia pode ser reduzido com insulinização adequada, como sugerem os dados do Diabetes Control and Complications Trial (DCCT). Com efeito, durante o transcorrer desse estudo, a incidência dessa complicação diminuiu substancialmente (4-6).

Embora a recuperação da função cerebral, em geral, seja completa após coma hipoglicêmico, pode haver seqüela permanente, com déficit neurológico significativo nas crianças em idade pré-escolar (7).

\section{DEFESA CONTRA HIPOGLICEMIA: MECANISMOS DE CONTRA- REGULAÇÃO EM NORMAIS}

A hipoglicemia deflagra uma série de mecanismos contra-reguladores: suprime a secreção de insulina pelas células-beta, estimula a liberação de glucagon pelas células-alfa, a de adrenalina pela medula adrenal, além do cortisol e do hormônio de crescimento. Além disso, observam-se também a liberação de noradrenalina de neurônios simpáticos pós-ganglionares e acetilcolina dos pós-ganglionares simpáticos e parassimpáticos, além de outros neuropeptídeos (8).

A queda da secreção de insulina possibilita aumentar a produção hepática e renal de glicose, além de diminuir sua captação nos tecidos periféricos, especialmente muscular.

O glucagon tem papel fundamental nesse mecanismo, aumentando a glicogenólise hepática e favorecen- do a gliconeogênese. A liberação de adrenalina resulta maior produção hepática de glicose e diminuição de captação nos tecidos insulino-sensíveis, além de ajudar na percepção dos sintomas e contribuir para diminuição de secreção de insulina por mecanismo $\alpha$-adrenérgico. Seu papel torna-se crítico quando a secreção de glucagon é insuficiente.

Os sintomas de hipoglicemia podem ser classificados em duas categorias: os neurogênicos ou autonômicos e os neuroglicopênicos.

Os primeiros são o resultado da percepção dos efeitos da liberação, mediada pelo sistema nervoso central (SNC), do sistema simpático/adrenal. Incluem sintomas adrenérgico (palpitação, tremor, ansiedade), mediados por adrenalina ou noradrenalina, e sintomas colinérgicos como sudorese, fome, parestesias, mediados por acetilcolina. A administração de antagonistas catecolaminérgicos e de acetilcolina bloqueia intensamente os sintomas de hipoglicemia, e é prudente que, se sua utilização pelos portadores de DMl for necessária, o médico deve estar atento para a possibilidade de agravamento de hipoglicemia assintomática.

Estudos em humanos adrenalectomizados mostram que a contribuição do sistema nervoso simpático neural é mais importante que a da medula adrenal, no que se refere aos sintomas, mas as alterações hemodinâmicas, como aumento de freqüência cardíaca e queda da pressão diastólica, são relacionados à adrenalina liberada de medula adrenal (9).

Os sintomas neuroglicopênicos refletem a disfunção provocada no SNC pela baixa concentração de glicose (Tabela 1).

Cortisol e hormônio de crescimento, cujas concentrações aumentam em resposta à hipoglicemia, limitam a utilização de glicose pelos tecidos periféricos. Esses hormônios têm um papel modesto na resposta à hipoglicemia aguda e são mais importantes quando esta é prolongada.

A liberação de ACTH, cortisol e ativação de sistema nervoso simpático decorrem de mediação de $\mathrm{SNC}$; o núcleo ventromedial hipotalâmico tem neurônios sensíveis à concentração de glicose que desencadeiam as respostas contra-reguladoras, embora outras regiões no SNC possam também ter algum papel.

Os mecanismos contra-reguladores são desencadeados em pessoas normais de maneira bastante reprodutível (Figura 1). 
Tabela 1. Sinais e sintomas de hipoglicemia.

\begin{tabular}{|l|l|}
\hline $\begin{array}{l}\text { Sintomas neuroautonômicos (causados pela resposta } \\
\text { autonômica) }\end{array}$ & $\begin{array}{l}\text { Sintomas neuroglicopênicos (causados pela concentração } \\
\text { reduzida de glicose no SNC) }\end{array}$ \\
\hline Falta de estabilidade, tremor & $\begin{array}{l}\text { Sensações estranhas (calor, formigamentos) } \\
\text { Irritabilidade } \\
\text { Ansiedade }\end{array}$ \\
Nervosismo & Dificuldado mental de raciocínio \\
Palpitações & Dificuldade de fala \\
Sudorese & Visão borrada \\
Pele pegajosa & Ataxia \\
Boca seca & Parestesias \\
Fome & Dor de cabeça \\
Palidez & Confusão mental \\
Dilatação da pupila & Convulsão \\
& Coma \\
& Morte \\
\hline
\end{tabular}

Morte cerebral

Figura 1. Limiares glicêmicos (sangue venoso arterializado) para os diversos contra-reguladores e sintomas.

Entretanto, esses limiares são dinâmicos e dependentes, sobretudo, do controle glicêmico prévio. Assim, pessoas com diabetes e controle glicêmico ruim, apresentando concentrações de hemoglobina glicada elevadas percebem sintomas de hipoglicemia quando as concentrações de glicose são mais altas do que em períodos de bom controle. O oposto é visto em portadores de diabetes tratados intensivamente que não reconhecem que estão em hipoglicemia até que os valores glicêmicos sejam extremamente baixos, e esse fenômeno agrava-se em relação às hipoglicemias prévias (10).
Defeito contra-regulatório na ativação simpática em resposta à hipoglicemia prévia não é exclusivo do estado diabético, como demonstrado também em normais: um único episódio de hipoglicemia causa diminuição significativa da resposta contra-reguladora a uma hipoglicemia subseqüente, o que pode ser um importante fator causal de hipoglicemia iatrogênica, sem sinais de alarme, apresentado por pessoas com DMl, fenômeno que passou a ser nomeado como hipoglicemia associada à insuficiência autonômica $(11,12)$.

\section{DEFESA CONTRA A HIPOGLICEMIA: MECANISMOS DE CONTRA-REGULAÇÃO NO DM1}

Em portadores de $\mathrm{DMl}$, obviamente, não ocorre diminuição da secreção de insulina em resposta à hipoglicemia, uma vez que sua concentração circulante, causadora de hipoglicemia, depende da absorção da insulina administrada.

A resposta esperada, de aumento na secreção de glucagon na vigência de hipoglicemia, deixa de existir precocemente no curso da doença em portadores de $\operatorname{DMl}(13)$.

Várias hipóteses tentam explicar esse evento: alteração na arquitetura da ilhota (14), neuropatia autonômi- 
ca (15), alteração no receptor de glicose na célula-alfa (16). No entanto, embora esses fatores possam contribuir, certamente não pode haver um fator único: assim, sabe-se que há liberação de glucagon a outros estímulos em portadores de $\mathrm{DMl}$, o que sugere um problema com a sinalização e não da estrutura da célula-alfa (17).

Estudos de denervação pancreática, assim como o bloqueio autonômico com antagonistas colinérgicos e adrenérgicos, mostram que após hipoglicemia existe liberação de glucagon. Finalmente, células-alfa viáveis, porém isoladas em cultura, não liberam glucagon após hipoglicemia, indicando que essa resposta não depende apenas da glico-regulação.

Estudos em humanos, com o uso de somatostatina ou de diazóxido, comprovam a teoria de que a diminuição da secreção da insulina intra-ilhota é um sinal importante para a liberação de glucagon na vigência da hipoglicemia. Em indivíduos normais, dois estímulos são necessários para a liberação desse hormônio: a diminuição de concentração de glicose na célula-alfa e a diminuição de secreção de insulina intra-ilhota (18).

A adrenalina e o glucagon estimulam a glicogenólise e a gliconeogênese, e a interação entre a metabolização do glicogênio e a gliconeogênese, em harmonia, possibilita rápida recuperação de hipoglicemia. Estudo recente, analisando a produção hepática de glicose e medida de glicogênio hepático por espectroscopia, verificou que, com efeito, em portadores de DMl, tratados intensivamente, a produção endógena de glicose diminui principalmente por glicogenólise deficiente (19).

Alguns estudos mostraram, além disso, diminuição do conteúdo hepático de glicogênio em diabéticos tipo l com controle intensivo, o que poderia contribuir para o agravamento de hipoglicemia. Estudos em humanos sugerem que a produção de glicose pelo fígado e pelos rins, em resposta à hipoglicemia, obedece a certa reciprocidade. Assim, por exemplo, diabéticos tipo 2 têm maior produção renal de glicose após hipoglicemia, compensando a deficiente produção hepática, explicando, parcialmente, ao menos, a baixa ocorrência de hipoglicemia em DM2. No DM1, no entanto, estão, diminuídas, após hipoglicemia, tanto a produção de glicose hepática quanto a renal $(20,21)$.

\section{HIPOGLICEMIA ASSOCIADA À INSUFICIÊNCIA AUTONÔMICA}

Em portadores de DMl, com mais de cinco anos de duração, a resposta de adrenalina, a principal arma de defesa contra a hipoglicemia, está freqüentemente atenuada, tendo o limiar para sua liberação mais baixo que em normais, especialmente após uma hipoglicemia prévia. Ainda mais, a magnitude da hipoglicemia sendo, habitualmente, precedente proporcional ao grau do bloqueio da resposta contra-reguladora. Dois episódios de glicemia menor que $70 \mathrm{mg} / \mathrm{dL}$ são suficientes para bloquear resposta contra-regulatória subseqüente em $30 \%$ (22). Em conseqüência, portadores de diabetes com resposta deficiente de glucagon e epinefrina têm risco 25 vezes maior de apresentar hipoglicemia quando em tratamento intensivo que os deficientes apenas de glucacon. Portanto, a falta dos sintomas de alarme, comprometendo a defesa comportamental contra a hipoglicemia (o ato de comer) está associada a maior risco de hipoglicemia grave (23).

\section{Hipoglicemia sem sinais de alarme}

A ocorrência de hipoglicemia sem sinais de alarme (bypoglycemic anawareness) foi descrita há mais de 50 anos em pessoas com DMl e também em portadores de insulinoma, que toleram concentrações glicêmicas muitíssimo baixas sem os sintomas de alarme que permitem que o paciente reconheça o episódio e tome as providências adequadas (comer) para abortá-lo. Sabe-se hoje que esse fenômeno não é uma questão de tudo ou nada: há um amplo espectro de apresentação, desde a total ausência de alarme até a presença de sintomas discretos ou atípicos. Pensou-se que esse fenômeno fosse uma complicação permanente relacionado à neuropatia autonômica, mas sabe-se que ele pode ser revertido se o paciente conseguir permanecer rigorosamente sem nenhuma hipoglicemia por um tempo variável de algumas semanas a poucos meses.

A insuficiência autonômica associada à hipoglicemia deve ser diferenciada da neuropatia autonômica clássica. Os portadores desta complicação podem ter risco maior de hipoglicemia grave pela falta de alarme adrenérgico (24). Insuficiência autonômica relacionada à hipoglicemia, no entanto, é reversível, evitando-se meticulosamente episódios de hipoglicemia.

Uma hipótese para explicar a diferença nos limiares de liberação de contra-reguladores é a de que a eficiência dos transportadores de glicose para o SNC tem relação com o controle glicêmico prévio. Em animais, após alguns dias de hipoglicemia freqüente, $\mathrm{o}$ transporte de glicose para o SNC aumenta, assim como a expressão de RNA-mensageiro do GLUTl e de sua proteína (25). O transporte aumentado de glicose para 
o SNC não se comprovou em humanos, utilizando-se tomografia com emissão de pósitrons para quantificar a metabolização de glicose; o fluxo cerebral também não se mostrou alterado em portadores de DMl (26). No entanto, recentemente, estudo da cinética cerebral de glicose utilizando PET-FDG (tomografia por emissão de pósitron usando fluorodeoxiglicose) em dois grupos de diabéticos tipo 1 , diferentes quanto à percepção de glicemia (um dos grupos não tinha sinais de alerta), mostrou que há diferenças importantes entre eles no que se refere à ativação de regiões cerebrais relacionadas com a resposta neuro-humoral e também de regiões associadas à percepção de emoções. Portadores de diabetes e hipoglicemia sem alarme têm ativação reduzida de regiões envolvidas na interocepção e no reconhecimento do valor do alimento e menor desativação de percepções prazerosas $(27,28)$. Isto explica parcialmente porque, às vezes, é tão difícil reverter o quadro de hipoglicemia sem sinais de alarme. O conceito de insuficiência autonômica associada à hipoglicemia foi ampliadorecentemente, incluindo também as hipoglicemias relacionadas ao exercício e ao sono.

\section{Hipoglicemia do exercício associada à insuficiência autonômica}

Em humanos normais, exercício físico moderado e prolongado diminui significativamente a resposta neuroendócrina e metabólica à hipoglicemia subseqüente. Entre outros efeitos, diminui a liberação de adrenalina em cerca de $30 \%$, o que torna a percepção de hipoglicemia mais difícil (29).

A hipoglicemia ocorre durante o exercício, em diabéticos em insulinoterapia, nas primeiras horas após seu término e até mesmo aproximadamente 20 horas depois. Hipoglicemia durante o exercício explica-se por maior consumo de glicose muscular, maior absorção de insulina nos locais de aplicação e defeitos na contra-regulação. Hipoglicemia tardia -6 a 20 ou mais horas após o exercício - resulta também desses mesmos mecanismos, além da melhora de sensibilidade à insulina. É importante acentuar que o risco de hipoglicemia tardia pode ser decorrente da contra-regulação alterada, uma vez que exercício prévio diminui a resposta de glucagon, noradrenalina e adrenalina à hipoglicemia subseqüente (30).

A mudança de dose de insulina para adequação ao exercício deve levar em consideração o tipo, a intensidade e a duração do exercício. Em nosso serviço, no Hospital das clínicas da Faculdade de Medicina da Universidade de São Paulo (FMUSP), recomenda-se que quando o portador de diabetes faz exercícios programados de $\mathrm{l}$ a 3 horas após a alimentação, a dose de insulina prandial deve ser reduzida pela metade, e, se necessária, ainda maior. Às vezes, é necessária a redução de $\mathrm{l}$ a 2 unidades da insulina basal anterior. Se o exercício é feito por mais de 3 horas após a alimentação, ou se o portador de DMl se exercita de maneira não programada, ou se a glicemia for menor que $100 \mathrm{mg} / \mathrm{dL}$, recomenda-se a ingestão adicional de 15 a 30 gramas de hidrato de carbono.

Um estudo neste hospital, que comparou exercícios aeróbicos e resistidos em DMl, com as modificações no controle referidas anteriormente, não observou hipoglicemia grave ou aumento da freqüência de hipoglicemia noturna sintomática (dados não publicados).

\section{Hipoglicemia durante o sono associada à insuficiência autonômica}

A hipoglicemia iatrogênica acontece com freqüência durante o sono, especialmente quando a insulina de ação intermediária com pico é prescrita antes do jantar. No Diabetes Control and Complications Trial (DCCT), $55 \%$ dos episódios de hipoglicemia grave ocorreram durante o sono, sendo $43 \%$ deles entre meia-noite e 8 horas da manhã (31).

Além de ser um período de maior risco, por conta de maior tempo sem alimento e de maior sensibilidade à insulina, demonstrou-se que as respostas de adrenalina e noradrenalina são menores, em DMl, após hipoglicemia durante o sono do que no mesmo paciente durante a vigília (32).

Além disso, portadores de $\mathrm{DMl}$, comparados a controles, têm maior dificuldade de despertar durante a hipoglicemia pelo fato de apresentarem menor liberação de adrenalina e noradrenalina $(33,34)$.

As implicações desse fato para a qualidade de vida de diabéticos e seus familiares são bem conhecidas. Não é incomum que as mães passem a dormir ao lado de seus filhos com medo das hipoglicemias noturnas. Com efeito, mostrou-se que o controle glicêmico de crianças pequenas em uso de infusão contínua de insulina é inversamente proporcional ao medo que seus pais têm de hipoglicemia (35).

\section{Efeito Somogyi}

Em 1959, Michael Somogyi relatou que hipoglicemias durante a noite eram seguidas por glicosúria maciça na manhã seguinte (36). Com os anos, prevaleceu o conceito 
de que a liberação de hormônios contra-reguladores após hipoglicemia era de magnitude suficiente para provocar hiperglicemia reacional. Embora estudos mensurando glicemia plasmática tenham já negado a existência desse fenômeno, a medida contínua de glicemia intersticial (continuous glucose monitoring system - CGMS) trouxe a confirmação da inexistência de hiperglicemia após hipoglicemia (37). Høi-Hansen e cols. estudaram 126 portadores de DMl durante seis noites seguidas perfazendo 756 noites. Em 23\% delas, valores de glicemia intersticial menores que $40 \mathrm{mg} / \mathrm{dL}$ ocorreram com duração maior que dez minutos. A glicemia na manhã seguinte foi cerca de $100 \mathrm{mg} / \mathrm{dL}$ menor nos pacientes que tiveram hipoglicemia de madrugada que a dos que se mantiveram euglicêmicos ou hiperglicêmicos durante a noite (38).

Outros estudos com o CGMS confirmam que as hipoglicemias noturnas são extremamente freqüentes. Guillod e cols. observaram que sua prevalência era de $67 \%$, sendo $32 \%$ não suspeitada, sugerindo que a medida de glicemia capilar ao se deitar tem sensibilidade de detectar hipoglicemia igual a da medida feita às 3 horas da manhã, além de mostrar que o CGMS é muito útil para a detecção e posterior diminuição de hipoglicemias noturnas (39).

\section{FATORES AGRAVANTES}

$\mathrm{Na}$ insuficiência renal, pacientes pouco dispostos a aderir aos esquemas de tratamento e com capacidade limitada de compreensão da farmacocinética de insulinas em uso têm risco muito maior de hipoglicemia. Várias medicações, em especial drogas psicoativas e betabloqueadores, podem diminuir a intensidade dos sintomas de hipoglicemia, contribuindo para o estabelecimento de hipoglicemia sem alerta.

Os antagonistas beta-adrenérgicos (betabloqueadores) teriam, teoricamente, poder de atenuar os sintomas de hipoglicemia e efeitos menos evidentes com os cardiosseletivos.

Alguns relatos de casos isolados mostraram o desaparecimento dos sintomas de alarme à hipoglicemia com o uso de inibidores seletivos da recaptação de serotonina, tendo como resultado hipoglicemia grave; o mecanismo é desconhecido, podendo ser uma apresentação atípica de disfunção autonômica. Importante também é o efeito do álcool, que pode ser imediato ou tardio, ocorrendo em especial após o café-da-manhã, posterior a seu uso (40).
Quando além da deficiência de glucagon e adrenalina se sobrepõe a de GH e cortisol, o risco de hipoglicemia aumenta acentuadamente, sendo necessária a modificação das doses de insulinas basal e prandial. Considerando-se que a hipofisite linfocítica crônica, embora muito rara, pode acometer portadores de DMl mais que a população geral, entende-se que a avaliação da função adrenal e de GH deva ser feita quando a freqüência de hipoglicemia parecer inexplicável (41).

O tipo de tratamento instituído pode aumentar o risco de hipoglicemia sem alarme. Em nossa experiência, o uso de insulina de ação intermediária em uma ou duas doses diárias, nas proporções dois terços pela manhã e um terço antes do jantar, freqüente no passado, é a principal causa de hipoglicemias repetidas, com ou sem sintomas (Figura 2).

A administração de insulina de forma mais fisiológica, basal-prandial, diminui o risco de hipoglicemia. Comparando-se análogos de insulina (de ação prolongada e ultra-rápida) às insulinas humanas (NPH e regular) observou-se discreta diminuição na freqüência de hipoglicemia, especialmente noturna, com os aná$\log 0 \mathrm{~s}(42)$.

\section{COMO DEFINIR E QUANTIFICAR A HIPOGLICEMIA}

Embora pareça óbvio que a definição de hipoglicemia devesse apontar para um número $(70 \mathrm{mg} / \mathrm{dL}$, valor a

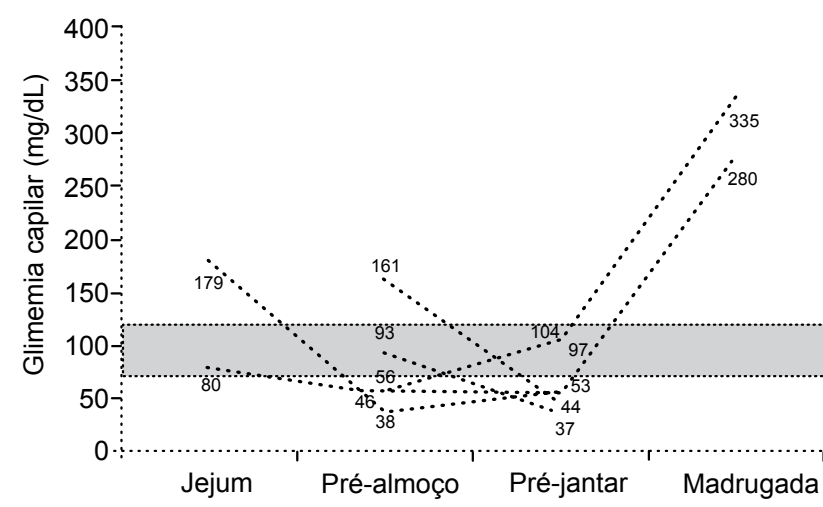

Figura 2. Monitoração glicêmica domiciliar de portador de DM1 em uso de insulina NPH: 38 unidades pela manhã e 14 ao se deitar, mostrando inúmeras hipoglicemias, sem nenhum sintoma e $\mathrm{HbAlc}$ de $7,3 \%$. 
partir do qual os hormônios contra-reguladores são liberados), os portadores de diabetes têm sintomas sob concentrações glicêmicas diversas. Além disso, grande parte das hipoglicemias percebidas e tratadas no dia-adia não chegam a ser medidas ou registradas. Por esse motivo, o grupo de trabalho da Associação Americana de Diabetes (43) propõe classificar hipoglicemia em:

- hipoglicemia grave (severe hypoglycemia): evento que requer assistência de outra pessoa para administração de carboidrato, glucagon, glicose oral ou endovenosa. Implica neuroglicopenia suficiente para induzir convulsão, alteração de comportamento ou coma. A medida da glicemia não é obrigatória no momento da hipoglicemia para classificá-la retrospectivamente como tal. A recuperação neurológica é considerada evidência suficiente;

- hipoglicemia sintomática documentada: evento com sintomas e glicemia menor que $70 \mathrm{mg} / \mathrm{dL}$;

- hipoglicemia assintomática (sem alarme): evento sem sintomas típicos de hipoglicemia, mas com a medida inferior a $70 \mathrm{mg} / \mathrm{dL}$;

- hipoglicemia sintomática provável: evento no qual os sintomas não são confirmados por medida de glicemia. Esses são eventos dificilmente quantificáveis em estudos clínicos;

- hipoglicemia relativa: evento no qual a pessoa portadora de diabetes atribuídos à hipoglicemia, mas com medida superior a $70 \mathrm{mg} / \mathrm{dL}$. Isto ocorre quando o controle glicêmico é inadequado.

Não há, até o momento, padrão para quantificar hipoglicemia. Recomenda-se que, em estudos clínicos, a freqüência de eventos seja relatada, discriminando as hipoglicemias noturnas das diurnas, pela diferença no risco entre elas, e também as características da hipoglicemia.

O grupo de trabalho considera que diminuir a incidência de hipoglicemia grave ainda que em $10 \%$ a $20 \%$ seja bastante vantajoso. A redução de hipoglicemia documentada (com ou sem sintoma) em mais de 30\% após a introdução de novo medicamento, monitoração ou educação, representaria um êxito considerável.

É importante considerar que, ao atribuir a um novo medicamento ou insumo um papel na redução de risco de hipoglicemia, deve-se ponderar que a diminuição da freqüência e da intensidade das hipoglicemias não deveria acompanhar-se de aumento de hemoglobina glicada. Na prática clínica, é quase sempre o que acontece. As hipoglicemias assintomáticas quando melhoram seguem-se freqüentemente de elevação da $\mathrm{HbAlc}$ (43).

\section{HIPOGLICEMIA E ACIDENTES AUTOMOBILÍSTICOS}

Entre todas as dificuldades e os constrangimentos enfrentados pelas pessoas portadoras de diabetes em razão de hipoglicemias, uma das mais significativas é a relacionada ao risco de acidentes automobilísticos, que podem afetar não só o paciente como outras pessoas. Um estudo que investigou a história médica de acidentes de trânsito não mostrou diferença no risco de hipoglicemia se a terapêutica era intensiva ou convencional, mas era muito maior quando, nos seis meses precedentes, o paciente tinha sofrido um episódio de hipoglicemia grave (44). No DCCT, acidente de trânsito com dano para propriedade ocorreu em 1,5\% dos 714 episódios de hipoglicemia grave.

Os portadores de diabetes com hipoglicemia sem alarme também não têm muito claro o risco que correm ao dirigir. Stork e cols. mostraram que, em experimentos em euglicemia ou em hipoglicemia, $43 \%$ dos portadores de diabetes tipo 1 com hipoglicemia sem alarme tomavam decisão de dirigir em condições hipoglicêmicas, enquanto isso ocorria em $4 \%$ dos diabéticos tipo 1 que apresentam sintomas adrenérgicos habituais (45).

Apesar disso, a freqüência de acidentes provocados por portadores de diabetes é controversa, havendo relatos de freqüência maior, igual e mesmo menor do que na população geral, embora estudos mais recentes apontem que há um pequeno aumento de risco de acidentes de trânsito quando o motorista é diabético. Indiscutivelmente, o risco é principalmente relacionado à hipoglicemia durante a viagem (3).

Eadington e cols. observaram que nove de 272 episódios $(3,3 \%)$ de hipoglicemia leve ou grave, que ocorreram enquanto o portador de diabetes estava dirigindo, resultaram acidentes automobilísticos (46).

A orientação para a direção defensiva deve ser sempre praticada pela equipe multidisciplinar. Portadores de diabetes em insulinoterapia devem fazer a medida de glicemia capilar antes de dirigir e repetir o procedimento a cada três ou quatro horas, se a viagem for de longa distância, tendo sempre à disposição, além do monitor, carboidratos de absorção rápida e de fácil ingestão. A glicemia capilar menor do que $70 \mathrm{mg} / \mathrm{dL}$ contra-indica 
a direção. Quando essa medida está entre 70 e $90 \mathrm{mg} / \mathrm{dL}$, é recomendável a ingestão de carboidrato, e não se deve dirigir nos 45 a 60 minutos subseqüentes à hipoglicemia leve a moderada.

Pessoas com hipoglicemia sem sintomas de alarme devem medir a glicemia capilar mais freqüentemente, além de elevar o alvo glicêmico, sendo prudente não ter glicemias capilares menores que $100 \mathrm{mg} / \mathrm{dL}$. Esse alvo glicêmico é válido também para outras pessoas ou situações nas quais o risco de hipoglicemia seja muito alto: crianças abaixo de 5 anos, idosos, pessoas com profissões de risco.

\section{HIPOGLICEMIA AGUDA E LESÃO CEREBRAL}

À medida que a glicemia cai, sinais clínicos de disfunção cerebral vão aparecendo, acompanhados por profundas alterações nas sinapses cerebrais, na neurotransmissão de monoaminas e também eletroencefalográficas. A atividade elétrica, no início da hipoglicemia, passa a mostrar lentificação e aumento de amplitude; quando mais grave, a atividade paroxística fica evidente e evolui; quando as concentrações glicêmicas estão muito baixas (inferiores a $18 \mathrm{mg} / \mathrm{dL}$ ) para a inatividade elétrica cerebral, e o EEG fica isoelétrico. Cerca de dez minutos após infusão de glicose, inicia-se a recuperação do padrão elétrico (47).

Há características importantes que diferenciam a lesão hipoglicêmica da anóxia. Na primeira, há aumento de produção de amônia e diminuição de lactato, com aumento do $\mathrm{pH}$, e, em consequiência, não ocorre infarto no parênquima cerebral. Um dos mistérios da lesão hipoglicêmica é o fato de ela ser freqüentemente assimétrica. Isso acontece talvez porque, por alterações na circulação sanguínea entre os hemisférios, a instalação de EEG isoelétrico não é simultânea entre diferentes localizações cerebrais, o que explica a ocorrência de sinais de localização por hipoglicemia.

Além disso, algumas áreas cerebrais são mais susceptíveis a lesão hipoglicêmica do que outras - classicamente o giro dentado, no hipocampo, é acometido precocemente, e o cerebelo é mais resistente (48).

Recentemente, Suh e cols. (47) demonstraram, em animais, que a morte dos neurônios está relacionada a vários mecanismos que incluem a liberação de glutamato e a ativação de seus receptores de produção de espé- cies reativas de oxigênio, liberação neuronal de zinco, ativação de poli (ADP-ribose) polimerase. Estudos de hipoglicemia seguida por administração de glicose mostram que a produção de superóxido e morte neuronal são aumentadas pela ativação de NADPH oxidase durante a reperfusão de glicose, e é maior após hiperglicemia induzida pela administração de glicose do que mantendo-se o animal em hipoglicemia grave. Não está claro se esses dados podem ser extrapolados para a prática clínica. Como os autores apontam, a glicemia deve ser corrigida, após um coma hipoglicêmico o mais rapidamente possível para se evitar a morte dos neurônios; no entanto, os valores glicêmicos a serem atingidos deveriam ser fisiológicos. Até o momento, não há evidência de que a hiperglicemia intensa após hipoglicemia seja maléfica para a recuperação, embora o bom senso diga que não há motivo para ser benéfica (49).

Assim, em coma hipoglicêmico profundo e prolongado, a elevação da glicemia deve ser feita cuidadosamente, mantendo-a em valores normais. Isto não se aplica às hipoglicemias moderadas ou leves.

A hipoglicemia aguda certamente altera, temporariamente, a função cognitiva, quase sempre recuperando-se completamente. No entanto, não é claro se existe disfunção cognitiva induzida por hipoglicemias repetidas no decorrer da vida do portador de diabetes. O estudo EDIC/DCCT (Epidemiology ofDiabetes Interventions and Complications/Diabetes Control and Complications Trial) não verificou relação entre presença de hipoglicemias e alterações cognitivas; pelo contrário, essas alterações foram relacionadas aos valores elevados de hemoglobina glicada (50). Contudo, métodos de detectar disfunção cognitiva podem não ser suficientemente sensíveis.

Alguns estudos mostraram prejuízo de habilidade visoespacial e motora em pacientes com $\mathrm{DMl}$ e história de hipoglicemia recorrente (51).

Os estudos experimentais apontam que o risco de lesão por hipoglicemia é maior no cérebro em desenvolvimento (52). Estudos clínicos também têm sido mais enfáticos em apontar que hipoglicemias repetidas ou graves, acometendo crianças antes dos 5 a 7 anos de idade podem contribuir para disfunções cognitivas (53-56).

Análises de desenvolvimento de filhos de mães diabéticas submetidas às hipoglicemias graves ou numerosas durante a gestação são escassas, mas há relatos isolados de disfunção cognitiva associada à grave hipoglicemia materna (57). 


\section{TRATAMENTO}

Um dos principais problemas relacionados à tentativa de correção de hipoglicemia é o hipertratamento. Quando ocorre hipoglicemia, os sintomas são tão desagradáveis, por vezes tão angustiantes, que não é incomum que pessoas com diabetes passem a comer continuamente até ficarem assintomáticas. O resultado é a intensa hiperglicemia após o evento hipoglicêmico e instabilidade glicêmica.

O que o educador em diabetes tem de tornar claro é que:

- em média 15 gramas de carboidrato aumentam a glicemia em 40 a $50 \mathrm{mg} / \mathrm{dL}$;

- a absorção de carboidrato pelo trato gastrintestinal não é instantânea; por isso, recomenda-se a ingestão de 15 gramas de carboidrato simples e após 15 minutos repetir o procedimento, se necessário. Para pessoas muito ansiosas e que se hipercorrigem, uma medida educativa útil é medir a glicemia capilar após a correção: valores glicêmicos muito elevados podem ajudar a modificar, em um episódio posterior, a maneira intempestiva de reverter a hipoglicemia.

A reversão do quadro de hipoglicemia associada à insuficiência autonômica, com a volta dos sintomas de alarme pode ser bastante difícil. Até o momento, evitar hipoglicemia de maneira meticulosa e o treinamento para reconhecimento de hipoglicemias são as únicas maneiras de permitir que os sintomas adrenérgicos voltem a ocorrer (58).

Os derivados de metilxantina (teofilina e cafeína), em estudos com pequeno número de pacientes e por tempo limitado, mostraram que essas drogas foram capazes de melhorar os sintomas de hipoglicemia, levando a maior estado de alerta e aumentando a secreção de catecolaminas (59). O uso de terbutalina, um agonista $\beta_{2}$-adrenérgico, foi testado em pessoas com DMl, e teve como resultado melhora das hipoglicemias noturnas, porém à custa de hiperglicemia na manhã seguinte. Entretanto, não há estudos de longo prazo com nenhuma dessas drogas $(60,61)$.

A injeção de glucagon é um método eficiente de corrigir a hipoglicemia de diabéticos tipo 1 incapazes de se alimentar, em especial em situação de diminuição de capacidade cognitiva ou convulsão. Parentes do portador de diabetes com risco de hipoglicemia grave devem ser treinados para administrar glucagon por via subcutâ- nea, que tem a mesma eficácia que a intramuscular. Amam e cols. mostraram que administrando-se, a crianças, 10 ou $20 \mu \mathrm{g} / \mathrm{kg}$ de peso, a glicemia corrigia-se igualmente, elevando sua concentração após dez minutos e com persistência desse efeito por pelo menos uma hora $(62)$.

Recomenda-se a administração de glucagon na dose de $1 \mathrm{mg}$ para adultos e $0,5 \mathrm{mg}$ a crianças. Esse procedimento é extremamente seguro, tendo como efeito colateral principal náuseas, raramente vômitos, que correm 60 a 90 minutos após a injeção (63). Considerando-se que a única alternativa para o tratamento do $\mathrm{DMl} \mathrm{em}$ coma, que não o glucagon subcutâneo, é a administração de glicose endovenosa que só pode ser feita em ambiente hospitalar e que nas metrópoles brasileiras o acesso à unidade de emergência pode demorar um tempo prolongado, fica difícil entender por que tão poucos pacientes em nosso país são instruídos a receber essa prescrição, quando apropriada.

\section{REFERÊNCIAS}

1. McAulay V, Deary IJ, Frier BM. Symptoms of hypoglycaemia in people with diabetes. Diabet Med. 2001;18(9):690-705.

2. Cryer, PE. Glucose homeostasis and hypoglycemia. In: Larsen $\mathrm{PR}$, Kronenberg H, Melmed S, Polonsky K, editors. Williams textbook of endocrinology. 10.ed. Philadelphia (Pennsylvania): Saunders/Elsevier; 2003. p. 1585-618.

3. Begg IS, Yale JF, Houldern RL, Rowe RC, Canadian Diabetes Association's Clinical \& Scientific Section. Canadian Diabetes Association's Clinical Practice Guidelines for Diabetes and Private and Commercial Driving. Can J Diabetes. 2003;27: 128-40.

4. Egger M, Davey Smith G, Stettler C, Diem P. Risk of adverse effects of intensified treatment in insulin-dependent diabetes mellitus: a meta-analysis. Diabet Med. 1997;14(11):919-28.

5. The Diabetes Control and Complications Trial Research Group. The effect of intensive treatment of diabetes on the development and progression of long-term complications in insulindependent diabetes mellitus. N Eng J Med. 1993;329:977-86.

6. The Diabetes Control and Complications Trial Research Group. Hypoglycemia in the Diabetes Control and Complications Trial. Diabetes. 1997;46(2):271-86.

7. Agardh CD, Rosén I, Ryding E. Persistent vegetative state with high cerebral blood flow following profound hypoglycemia. Ann Neurol. 1983;14(4):482-6.

8. Ahrén B. Autonomic regulation of islet hormone secretion-implications for health and disease. Diabetologia. 2000;43(4):393-410.

9. DeRosa MA, Cryer PE. Hypoglycemia and the sympathoadrenal system: neurogenic symptoms are largely the result of sympathetic neural, rather than adrenomedullary, activation. Am J Physiol Endocrinol Metab. 2004;287(1):E32-41.

10. Boyle PJ, Schwartz NS, Shah SD, Clutter WE, Cryer PE. Plasma glucose concentrations at the onset of hypoglycemic symptoms in patients with poorly controlled diabetes and in nondiabetics. N Engl J Med. 1988;318:(23):1487-92. 
11. Heller SR, Cryer PE. Reduced neuroendocrine and symptomatic responses to subsequent hypoglycemia after 1 episode of hypoglycemia in nondiabetic humans. Diabetes. 1991;40(2):223-6.

12. Davis SN, Tate D. Effects of morning hypoglycemia on neuroendocrine and metabolic responses to subsequent afternoon hypoglycemia in normal man. J Clin Endocrinol Metab. 2001;86(5):2043-50.

13. Bolli G, de Feo P, Compagnucci P, Cartechini MG, Angeletti G, Santeusanio $F$, et al. Abnormal glucose counterregulation in insulin-dependent diabetes mellitus. Interaction of anti-insulin antibodies and impaired glucagon and epinephrine secretion. Diabetes. 1983;32:134-41.

14. Unger $\mathrm{RH}$. Insulin-glucagon relationships in the defense against hypoglycemia. Diabetes. 1983;2:575-83.

15. Taborsky GJ Jr, Ahren B, Mundinger TO, Mei Q, Havel PJ. Autonomic mechanism and defects in the glucagon response to insulin-induced hypoglycaemia. Diabetes Nutr Metab. 2002;15:318-22.

16. Gerich JE, Langlois M, Noacco C, Karam JH, Forsham PH. Lack of glucagon response to hypoglycemia in diabetes: evidence for an intrinsic pancreatic alpha cell defect. Science 1973;182(108):171-3.

17. Hoffman RP, Singer-Granick C, Drash AL, Becker DJ. Abnormal alpha cell hypoglycemic recognition in children with insulin dependent diabetes mellitus (IDDM). J Pediatr Endocrinol. 1994;7(3):225-34.

18. Gosmanov NR, Szoke E, Israelian Z, Smith T, Cryer PE, Gerich $\mathrm{JE}$, et al . Role of the decrement in intraislet insulin for the glucagon response to hypoglycemia in humans. Diabetes Care. 2005;28:1124-31.

19. Kishore P, Gabriely I, Cui MH, Di Vito J, Gajavelli S, Hwang JH, et al Role of hepatic glycogen breakdown in defective counterregulation of hypoglycemia in intensively treated type 1 diabetes. Diabetes. 2006;55(3):659-66.

20. Woerle HJ, Meyer C, Popa EM, Cryer PE, Gerich JE. Renal compensation for impaired hepatic glucose release during hypoglycemia in type 2 diabetes: further evidence for hepatorenal reciprocity. Diabetes. 2003;52(6):1386-92.

21. Cersosimo E, Garlick P, Ferretti J. Abnormal glucose handling by the kidney in response to hypoglycemia in type 1 diabetes. Diabetes. 2001;50(9):2087-93.

22. Davis SN, Shavers C, Mosqueda-Garcia R, Costa F. Effects of differing antecedent hypoglycemia on subsequent counterregulation in normal humans. Diabetes. 1997;46(8):1328-35.

23. Gold AE. Frequency of severe hypoglycemia in patients with type I diabetes with impaired awareness of hypoglycemia. Diabetes Care. 1994;17:697-703.

24. Stephenson JM, Kempler P, Cavallo Perin P, Fuller JH. Is autonomic neuropathy a risk factor for severe hypoglycemia? The EURODIAB IDDM Complications Study. Diabetologia. 1996;39(11):1372-6.

25. Kumagai AK, Kang YS, Boado RJ, Pardridge WM. Upregulation of blood-brain barrier GLUT1 glucose transporter protein and mRNA in experimental chronic hypoglycemia. Diabetes. 1995;44(12):1399-404.

26. Segel SA, Fanelli CG, Dence CS, Markham J, Videen TO, Paramore DS, et al. Blood-to-brain glucose transport, cerebral glucose metabolism, and cerebral blood flow are not increased after hypoglycemia. Diabetes. 2001;50(8):1911-7.

27. Dunn JT, Cranston I, Marsden PK, Amiel SA, Reed LJ. Attenuation of amydgala and frontal cortical responses to low blood glucose concentration in asymptomatic hypoglycemia in type
1 diabetes: a new player in hypoglycemia unawareness? Diabetes. 2007;56(11):2766-73.

28. Bingham EM, Dunn JT, Smith D, Sutcliffe-Goulden J, Reed LJ, Marsden PK, et al. Differential changes in brain glucose metabolism during hypoglycaemia accompany loss of hypoglycaemia awareness in men with type 1 diabetes mellitus. An [11C]-3-O-methyl-D-glucose PET study. Diabetologia. 2005;48 (10):2080-9.

29. Galassetti P, Mann S, Tate D, Neill RA, Costa F, Wasserman DH, et al. Effects of antecedent prolonged exercise on subsequent counterregulatory responses to hypoglycemia. Am J Physiol Endocrinol Metab. 2001;280(6):E908-17.

30. McMahon SK, Ferreira LD, Ratnam N, Davey RJ, Youngs LM, Davis $E A$, et al. Glucose requirements to maintain euglycemia after moderate-intensity afternoon exercise in adolescents with type 1 diabetes are increased in a biphasic manner. J Clin Endocr Metab. 2007;92(3):963-8.

31. The DCCT Research Group.Epidemiology of severe hypoglycemia in the Diabetes Control and Complications Trial. Am J Med. 1991;90:450-9.

32. Jones TW, Porter P, Sherwin RS, Davis EA, O'Leary P, Frazer F, et al . Decreased epinephrine responses to hypoglycemia during sleep. N Engl J Med. 1998;338(23):1657-62.

33. Schultes B, Jauch-Chara K, Gais S, Hallschmid M, Reiprich E, Kern W, et al. Defective awakening response to nocturnal hypoglycemia in patients with type 1 diabetes mellitus. PLoS Med. 2007;4:e69.

34. Banarer S, Cryer PE. Sleep-related hypoglycemia-associated autonomic failure in type 1 diabetes: reduced awakening from sleep during hypoglycemia. Diabetes. 2003;52:1195-203.

35. Patton SR, Dolan LM, Henry R, Powers SW. Parental fear of hypoglycemia: young children treated with continuous subcutaneous insulin infusion. Pediatr Diabetes. 2007;8:362-8.

36. Somogyi M. Exacerbation of diabetes by excess insulin action. Am J Med. 1959;26(2):169-91.

37. Hirsch IB, Smith LJ, Havlin CE, Shah SD, Clutter WE, Cryer PE. Failure of nocturnal hypoglycemia to cause daytime hyperglycemia in patients with IDDM. Diabetes Care. 1990;13(2):133-42.

38. Høi-Hansen T, Pedersen-Bjergaard U, Thorsteinsson B. The Somogyi phenomenon revisited using continuous glucose monitoring in daily life. Diabetologia. 2005;48:2437-8.

39. Guillod L, Comte-Perret S, Monbaron D, Gaillard RC, Ruiz J. Nocturnal hypoglycaemias in type 1 diabetic patients: what can we learn with continuous glucose monitoring? Diabetes Metab. 2007;33:360-5.

40. Turner BC, Jenkins E, Kerr D, Sherwin RS, Cavan DA. The effect of evening alcohol consumption on next-morning glucose control in type 1 diabetes. Diabetes Care. 2001;24(11):1888-93.

41. Pestell RG, Best JD, Alford FP. Lymphocytic hypophysitis. The clinical spectrum of the disorder and evidence for an autoimmune pathogenesis. Clin Endocrinol. 1990;33:457-66.

42. Gough SC. A review of human and analogue insulin trials. Diabetes Res Clin Pract. 2007;77:1-15.

43. American Diabetes Association Workgroup on hypoglycemia Defining and reporting hypoglycemia in Diabetes. A report from the American Diabetes Association workgroup on hypoglycemia. Diabetes Care. 2005;28:1245-9.

44. Songer TJ, Lave LB, LaPorte RE. The risks of licensing persons with diabetes to drive trucks. Risk Anal. 1993;13(3):319-26.

45. Stork AD, van Haeften TW, Veneman TF. The decision not to drive during hypoglycemia in patients with type 1 and type 2 
diabetes according to hypoglycemia awareness. Diabetes Care. 2007;30(11):2822-6.

46. Eadington DW, Frier BM.Type 1 diabetes and driving experience: an eight-year cohort study. Diabet Med. 1989;6(2):137-41.

47. Suh SW, Aoyama K, Chen Y, Garnier P, Matsumori Y, Gum E, et al. Hypoglycemic Neuronal Death and Cognitive Impairment Are Prevented by Poly (ADP-Ribose) Polymerase Inhibitors Administered after Hypoglycemia. J Neurosci. 2003;23(33): 10681-90.

48. Auer RN. Hypoglycemic brain damage. Metab Brain Dis. 2004;19(3-4):169-75.

49. Suh SW, Gum ET, Hamby AM, Chan PH, Swanson RA. Hypoglycemic neuronal death is triggered by glucose reperfusion and activation of neuronal NADPH oxidase. J Clin Invest. 2007;117:910-8.

50. Diabetes Control and Complications Trial/Epidemiology of Diabetes Interventions and Complications Study Research Group, Jacobson AM, Musen G, Ryan CM, Silvers N, Cleary P, Waberski B, et.al. Long-term effect of diabetes and its treatment on cognitive function. N Engl J Med. 2007;356:1842-52.

51. Wredling R, Levander S, Adamson U, Lins PE. Permanent neuropsychological impairment after recurrent episodes of severe hypoglycaemia in man. Diabetologia. 1990;33(3):152-7.

52. Kim M, Yu ZX, Fredholm BB, Rivkees SA. Susceptibility of the developing brain to acute hypoglycemia involving $A 1$ adenosine receptor activation. Am J Physiol Endocrinol Metab. 2005;289(4):E562-9.

53. Hannonen R, Tupola S, Ahonen T, Riikonen R. Neurocognitive functioning in children with type-1 diabetes with and without episodes of severe hypoglycaemia. Dev Med Child Neurol. 2003;45(4):262-8.

54. Northam EA, Anderson PJ, Jacobs R, Hughes M, Warne GL, Werther GA. Neuropsychological profiles of children with type 1 diabetes 6 years after disease onset. Diabetes Care. 2001;24(9):1541-6.

55. Hershey T, Perantie DC, Warren SL, Zimmerman EC, Sadler M, White $\mathrm{NH}$. Frequency and timing of severe hypoglycemia affects spatial memory in children with type 1 diabetes. Diabetes Care. 2005;28(10):2372-7.

56. Ferguson SC, Blane A, Wardlaw J, Frier BM, Perros P, McCrimmon RJ, et al. Influence of an early-onset age of type 1 diabetes on cerebral structure and cognitive function. Diabetes Care. 2005;28(6):1431-7.

57. ter Braak EW, Evers IM, Willem Erkelens D, Visser GH. Maternal hypoglycemia during pregnancy in type 1 diabetes: maternal and fetal consequences. Diabetes Metab Res Rev. 2002;18(10):96-105.

58. Malerbi FEK, Matos MA. Blood glucose discrimination training: the role of internal and external cues. $J$ Health Psychol. 2001;6:229-40.

59. de Galan BE, Tack CJ, Lenders JW, Pasman JW, Elving LD, Russel FG, et al. Theophylline improves hypoglycemia unawareness in type 1 diabetes. Diabetes. 2002;51:790-6.

60. Raju B, Arbelaez AM, Breckenridge SM, Cryer PE. Nocturnal hypoglycemia in type 1 diabetes: an assessment of preventive bedtime treatments. Clin Endocrinol Metab. 2006;91(6): 2087-92.

61. White Jr JR. The contribution of Medications to hypoglycemia unawareness. Diabetes Spectr. 2007;20:77-80.

62. Amam J, Wranne L. Hypoglycaemia in childhood diabetes. Acta Paediatr Scand. 1988;77:548-53.

63. Pollack CV. Utility of glucagon in the emergency department. J Emerg Med. 1993;195-205.

\section{Endereço para correspondência:}

Marcia Nery

Hospital das Clínicas FMUSP

Av. Dr. Enéas Carvalho Aguiar, 255, $7^{\circ}$ andar, sala 7037

05403-000 - São Paulo, SP

Email: marcianery@hcnet.usp.br 